\title{
Detection of an Oseltamivir-Resistant Pandemic Influenza A/H1N1 Virus in the United Arab Emirates
}

\author{
Mubarak Alfaresi $^{a}$ Saif Albedwawi ${ }^{b} \quad$ Mohammed Hag-Ali ${ }^{c}$ \\ Departments of a Pathology and Laboratory Medicine, ${ }^{b}$ Infection Control and ' Public Health, \\ Zayed Military Hospital, Abu Dhabi, United Arab Emirates
}

\section{Key Words}

Pandemic A/H1N1 virus • Oseltamivir resistance •

Neuraminidase gene

\begin{abstract}
We report the identification of an influenza $\mathrm{A}(\mathrm{H} 1 \mathrm{~N} 1)$ viral isolate found to be resistant to oseltamivir. This is the first report of resistance of $\mathrm{H} 1 \mathrm{~N} 1$ to this medication in the United Arab Emirates. Sequencing of the isolate showed that it has the H275Y mutation.

Copyright $\odot 2010$ S. Karger AG, Base
\end{abstract}

\section{Introduction}

The emergence of influenza A pandemic (H1N1) 2009 virus, presumably from swine to humans, has spread globally since April 2009 [1]. This emergence prompted the World Health Organization (WHO) to declare a pandemic of this virus on June 11, 2009. Although most cases of infection are mild or asymptomatic, 15,174 fatal cases were reported to the WHO as of February 5, 2010 [2].

Therapeutic options are presently limited to two neuraminidase (NA) inhibitors, oseltamivir and zanamivir, because this virus has a swine origin matrix 2 (M2) gene, which contains a mutation associated with resistance to the M2 ion channel blockers amantadine and rimantadine. Although oseltamivir has been widely used in persons infected with pandemic (H1N1) 2009 virus, resistance was not observed until recently $[3,4]$.

Emergence of resistance to oseltamivir by seasonal influenza A (H1N1) virus was detected in Norway in 2007. This virus has evolved into the dominant influenza A virus (H1N1) in humans [3]. During the 2007-2008 influenza season, 264 of 268 influenza A (H1N1) viruses were reported to be resistant to oseltamivir [4]. Two screening tools are usually used to determine oseltamivir resistance: pyrosequence analysis of viral genes and an NA inhibition assay. As of September 1, 2009, a total of 2,974 influenza A (H1N1) samples were tested for oseltamivir resistance by the Centers for Disease Control and Prevention (CDC), of which 41 (1.4\%) samples were positive (CDC, 2010). This finding raises strong concerns that the H274Y resistant mutation in the pandemic (H1N1) 2009 virus might circulate and become dominant. We report the virologic investigation of the emergence of oseltamivir resistance in this virus in a patient from the United Arab Emirates (UAE).

\section{KARGER}

Fax +4161306 1234 E-Mail karger@karger.ch www.karger.com
(C) 2010 S. Karger AG, Base

1011-7571/11/0201-0097\$38.00/0

Accessible online at:

www.karger.com/mpp
Mubarak Alfaresi

Department of Pathology and Laboratory Medicine

Zayed Military Hospital, PO Box 3740

Abu Dhabi (United Arab Emirates)

Tel. +971 2405 5810, Fax +971 2449 2075, E-Mail uaenow@eim.ae 
Fig. 1. Phylogenetic analysis of the NA gene of pandemic influenza A/H1N1 viruses. Font in red indicates oseltamivirresistant virus A/UAE/A01/2009 (H1N1); classical swine $\mathrm{H} 1 \mathrm{~N} 1$ and seasonal $\mathrm{H} 1 \mathrm{~N} 1$ viruses are indicated in pink and green, respectively (colors in the online version only).

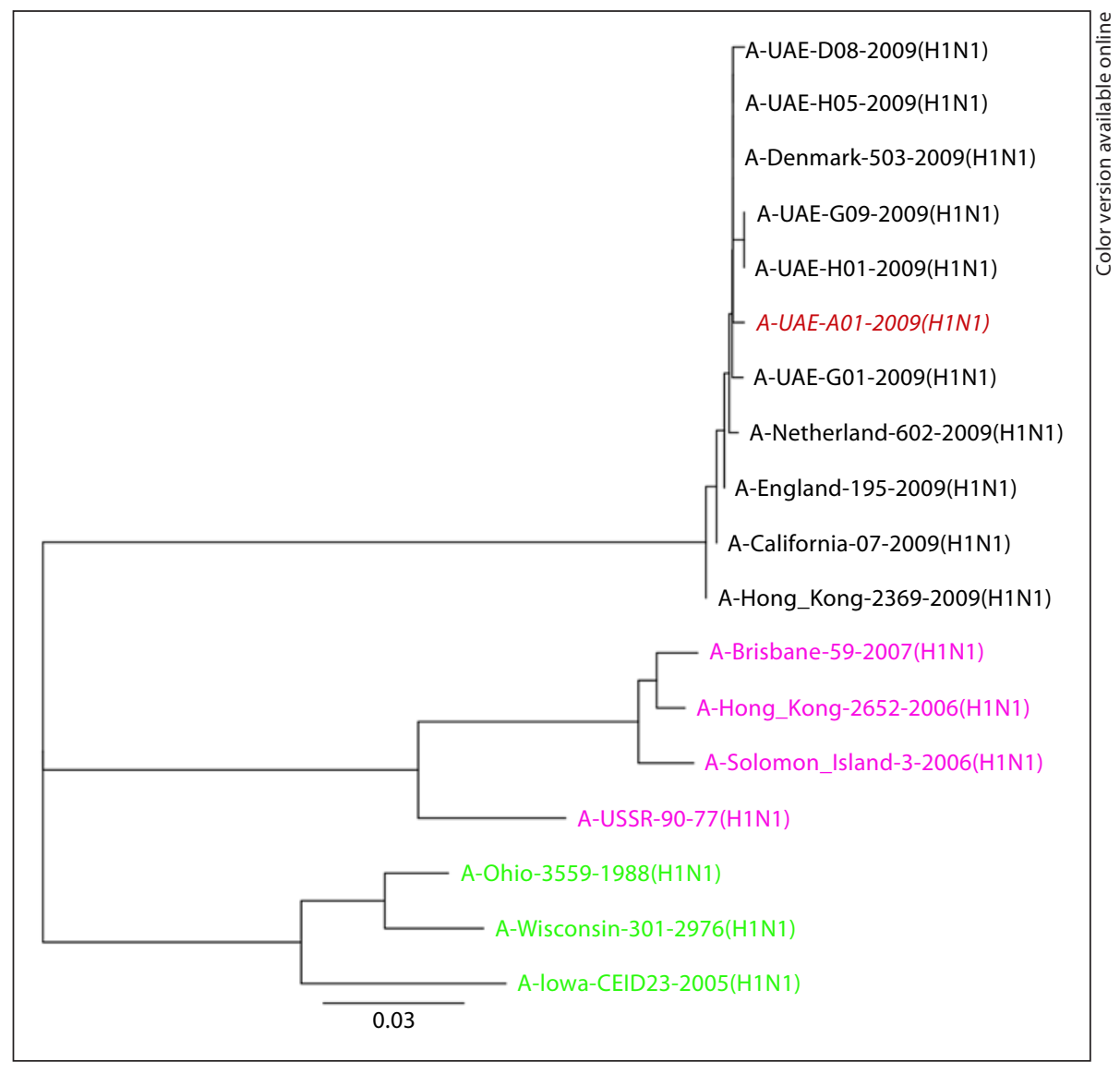

\section{Materials and Methods}

From July 1 to November 30, 2009, 96 respiratory specimens from cases positive for influenza A/H1N1 RT-PCR were tested for oseltamivir resistance. The testing was done by nucleotide sequencing of the NA gene by pyrosequencing using CDC primers Uni-sw-N1-B-F780, Uni-sw-N1-B-R1237-biot and Uni-sw-N1-BF804seq to detect the presence of the H275Y mutation as in the $\mathrm{WHO} / \mathrm{CD}$ pyrosequencing protocol [5].

\section{Results}

Of the 96 positive influenza $\mathrm{A} / \mathrm{H} 1 \mathrm{~N} 1$ isolates, we detected $1 \mathrm{~A} / \mathrm{H} 1 \mathrm{~N} 1$ strain which we designated as A/UAE/ A01/2009(H1N1) that was resistant to oseltamivir. The isolate was recovered from an 8-year-old child who had received a prophylactic course of treatment with oseltamivir because 2 of his siblings had confirmed H1N1 infection. Three days after finishing his prophylactic treatment, the child reported to the hospital with a characteristic clinical picture.
Sequences indicated that the NA genes in the nasopharyngeal aspirate isolate contained an $\mathrm{H} \rightarrow \mathrm{Y}$ mutation at the NA 274 (H3 numbering, 275 in $\mathrm{H} 1$ numbering) residue (Genbank accession No. CY053412). No other NA mutations known to be associated with oseltamivir resistance were observed.

\section{Discussion}

Resistance to NA inhibitors among seasonal strains of human influenza viruses (A/H1N1, A/H3N2 and B) has been rare until recently. Oseltamivir resistance associated with the NA 274Y genotype was also observed in human infections with avian influenza A virus (H5N1) [6].

Oseltamivir-resistant influenza A/H1N1 (2009) virus had been reported from Denmark, Japan and Hong Kong [7]. In all cases, however, the patients had a history of prior treatment with oseltamivir as in our patient which we attributed to the possibility of a drug-induced mutation. Development of resistance after oseltamivir treat- 
ment has occurred in $0.33-5.5 \%$ of treated patients. In our case, the nucleotide sequence was very similar to the circulating $\mathrm{A} / \mathrm{H} 1 \mathrm{~N} 1$ strains indicating that no reassortment with the NA gene of the oseltamivir-resistant $\mathrm{A} /$ Brisbane/59/2007-like clade 2B influenza type A (H1N1) had taken place (fig. 1).

We would like to point out that adequate knowledge of this virus is still limited, and characterization of trans- mission properties of this resistant variant in in vitro and in vivo models is needed. Moreover, pandemic (H1N1) 2009 virus should be closely monitored for emergence of resistant variants. It is imperative that systematic antiviral sensitivity testing should be undertaken to detect rapidly the emergence of antiviral resistance in both seasonal and pandemic influenza $\mathrm{A} / \mathrm{H} 1 \mathrm{~N} 1$ virus.

\section{References}

1 Chen H, Cheung CL, Tai H, Zhao P, Chan JF, Cheng VC, Chan KH, Yuen KY: Oseltamivirresistant influenza A pandemic (H1N1) 2009 virus, Hong Kong, China. Emerg Infect Dis 2009; 15:1970-1972.

2 World Health Organization H1N1 update. http://www.who.int/csr/don/2010_02_5/en/ index.html (accessed February 8, 2010).

3 Hauge SH, Dudman S, Borgen K, Lackenby A, Hungnes O: Oseltamivir-resistant influenza viruses A (H1N1), Norway, 2007-2008. Emerg Infect Dis 2009;15:155-162.
4 Dharan NJ, Gubareva LV, Meyer JJ, OkomoAdhiambo M, McClinton RC, Marshall SA, St George K, Epperson S, Brammer L, Klimov AI, Bresee JS, Fry AM: Infections with oseltamivir-resistant influenza A (H1N1) virus in the United States. JAMA 2009;301: 1034-1041.

5 World Health Organization: Protocol for antiviral susceptibility testing by pyrosequencing. http://who.int/csr/resources/publications/swineflu/pyrosequencing protocol/ en/ (accessed July 15, 2009).
6 6 Le QM, Kiso M, Someya K, Sakai YT, Nguyen TH, Nguyen KH, Yamada S, Muramoto Y, Horimoto T, Takada A, Goto H, Suzuki T, Suzuki Y, Kawaoka Y: Avian flu: isolation of drug-resistant H5N1 virus. Nature 2005;437: 1108 .

7 World Health Organization: Viruses resistant to oseltamivir (Tamiflu) identified. http://www.who.int/csr/disease/swineflu/ notes/h1n1_antiviral_resistance_20090708/ en/ (accessed February 8, 2010). 